\title{
Heat Shock Protein 27 (HSP27) As a Therapeutic Target in Ischemic Stroke and Neurodegenerative Disorders
}

\author{
HIDEKI SHIMURA*1), RYOTA TANAKA*2), TAKAO URABE*1), NOBUTAKA HATTORI*2) \\ *1) Department of Neurology, Juntendo University Urayasu Hospital, Chiba, Japan, *2) Department of Neurology, Juntendo \\ University Faculty of Medicine, Tokyo, Japan
}

\begin{abstract}
Heat shock protein 27 (HSP27) is a multidimensional protein that acts as a protein chaperone and an antioxidant and plays a role in the inhibition of apoptosis and actin cytoskeletal remodeling. In each of these capacities, HSP27 has been implicated in protective roles in various disease states. The current review presents HSP27 in multiple disease contexts, including neurodegenerative and cardiovascular diseases, highlighting its role as a potential therapeutic target.
\end{abstract}

Key words: Heat shock protein 27 (HSP27), stroke, therapy

Heat shock protein 27 (HSP27) (UniProt accession no. P04792) belongs to the small molecular weight heat shock protein (HSP) family (12-43 $\mathrm{kDa})^{1)}$, and is a human homologue of HSPB1. HSP27 is ubiquitously expressed in all human tissues with the highest level of expression in skeletal, smooth, and cardiac muscles ${ }^{2}$. HSP27 and other members of the small HSP family share a conserved c-terminal domain, the $\alpha$-crystallin domain, which is identical to the vertebrate eye lens $\alpha$-crystallin ${ }^{1)}$. HSP27 was initially characterized in response to heat shock ${ }^{3)}$ as a protein chaperone that facilitates the proper refolding of damaged proteins ${ }^{4)}$. Further studies revealed that the protein responds to cellular stress conditions other than heat shock, for example oxidative stress. During oxidative stress, HSP27 functions as an antioxidant, lowering the levels of reactive oxygen species (ROS) by raising levels of intracellular glutathione ${ }^{5)}$. The protein functions as an anti-apoptotic agent under conditions of chemical stress by interacting with both mitochondrial- dependent and independent pathways of apoptosis. HSP27 directly binds DAXX during Fas-FasLmediated apoptosis and prevents the subsequent binding of Ask1 by DAXX ${ }^{6}$. HSP27 also interacts with Bax and cytochrome c, thereby preventing mitochondrial-dependent apoptosis ${ }^{7)}$. HSP27 is particularly involved in protection from programmed cell death by inhibiting caspase-dependent apoptosis ${ }^{8)}$. HSP27 has also been characterized with the ability to regulate actin cytoskeletal dynamics during heat shock and other stress conditions, functioning both to promote actin polymerization and as an actin capping protein ${ }^{9}$. HSP27 is also found in the extracellular space where it may signal via membrane receptors to alter gene transcription and cellular function. Extracellular HSP27 is thought to be an immunomodulator ${ }^{10)}$. HSp27 has various cytoprotective functions and is thought that overexpression or replacement of HSP27 may ameliorate pathological lesions and symptoms of or cure various diseases ${ }^{1)}$. Overexpression was

Corresponding author: Hideki Shimura

Department of Neurology, Juntendo University Urayasu Hospital

2-1-1 Tomioka, Urayasu-shi, Chiba 279-0021, Japan

TEL: +81-47-353-3111 FAX: +81-47-353-3111 E-mail: miurashimura@yahoo.co.jp

339th Triannual Meeting of the Juntendo Medical Society: Medical Research Update〔Held on May 21, 2016〕

[Received Nov. 5, 2016]

Copyright (C) 2017 The Juntendo Medical Society. This is an open access article distributed under the terms of Creative Commons Attribution License (CC BY), which permits unrestricted use, distribution, and reproduction in any medium, provided the original source is properly credited. doi: $10.14789 /$ jmj. 63.17 
effective against ischemic brain disease, ischemic heart disease, ischemic renal disease, ischemic liver disease, Alzheimer's disease, and Parkinson's disease ${ }^{11}$. Here we discuss the possibilities of HSP27 therapy, including our own work.

\section{Neurodegenerative disease}

Neurodegenerative diseases are characterized by the accumulation of misfolded proteins. Alzheimer's disease is the most common neurodegenerative disorder. In neurons of patients with Alzheimer's disease, HSPB1 is colocalized with neurofibrillary tangles formed by phosphorylated tau ${ }^{12)}$ and senile plaques formed by amyloid $-\beta^{13)}$. Accumulation of phosphorylated tau is one major pathogenesis of Alzheimer's disease. In 2004, Shimura, et al. first showed the therapeutic potential of HSP27 in Alzheimer's disease. HSP27 delivered to human cortical neuron 2A cultured cells decreased phosphorylated tau ${ }^{14)}$. In 2010, Abisambra, et al. demonstrated the potential therapeutic properties of HSP27 in in vitro and in vivo models ${ }^{15}$. Overexpressed HSP27 and its constitutively phosphorylated mutant form both interacted with and prevented tau accumulation. Accumulation of amyloid- $\beta$ is another major pathogenesis of Alzheimer's disease. HSP27 also decreased the amount of aggregated amyloid- $\beta$ in a mouse model of Alzheimer's disease ${ }^{16)}$.

Parkinson's disease is the second most common neurodegenerative disorder. Accumulation of aggregated $\alpha$-synuclein is thought to be the major pathogenic process in Parkinson's disease. In neurons of patients with Alzheimer's and Parkinson's disease, HSPB1 is colocalized with Lewy bodies ${ }^{17)}{ }^{18)}$. Overexpressed HSP27 also decreased the amount of aggregated $\alpha$-synuclein in a mouse model of Parkinson's disease ${ }^{19)}{ }^{20)}$. HSP27 replacement therapy might help to decrease the pathological aggregations of amyloid- $\beta$, tau, and $\alpha$-synuclein and might be effective for two of the major neurodegenerative disorders, Alzheimer's disease and Parkinson's disease.

\section{Cerebrovascular disease}

Cerebral infarcts are characterized by pannecrosis, not only of neurons but also of glial cells and vascular elements, stemming from a disruption of the cerebral blood supply, which may cause permanent neurological damage, complications, and disabilities ${ }^{21}$. After focal ischemia, there are two different pathophysiologies of injury related to primary anoxic ischemic cell death and delayed secondary neuronal injury by reperfusion or reoxygenation ${ }^{22)}$. Secondary brain injury is characterized by adhesion molecules, leukocyte infiltration, and activation of immune cells (macrophages, microglia, and astroglia), and results in the overexpression of cytokines, such as IL- $1 \beta$, TNF- $\alpha$, and chemokines, within hours, leading to neuronal cell death ${ }^{23)}$. Kato, et $_{\text {al. }}{ }^{24)}$ demonstrated a time-dependent increased expression of HSP27 in surviving microglia and astrocytes following transient ischemia and reperfusion. To determine the function of HSP27 up-regulation following cerebral ischemia, Stetler, et $a{ }^{25)}$ designed transgenic mice that globally overexpressed HSP27. The study showed that HSP27 protected brain and inhibited ASK1dependent MKK4/JNK activation, upstream of the mitochondrial-dependent pathways of apoptosis. Thus, HSP27 overexpression has potential protective effects against cerebral ischemia and subsequent neuronal injury, implicating it as a potential therapeutic agent during stroke.

Recent studies showed that extracellular HSP27 plays a role as an immunomodulator and that extracellular HSP27 is transferred intracellularly by endocytosis and directly internalization through receptors ${ }^{10}$. We examined HSP27 replacement therapy by injecting HSP27 intravenously, as would be done clinically, and demonstrated that intravenously injected phosphorylated HSP27 protected the brain in transient middle cerebral artery occlusion (tMCAO) model mice ${ }^{25)}$, whereby it suppressed apoptotic cell death, oxidative DNA damage, and inflammatory responses, and also preserved the blood brain barrier. We first tested HSP27 purified from human lymphocytes (hHSP27) ${ }^{25}$. hHSP27 differed from recombinant HSP27 in that it formed dimeric, tetrameric, and multimeric complexes, was phosphorylated, and contained small amounts of $\alpha \mathrm{B}^{-}$-crystallin and HSP20. Mice received intravenous injections of hHSP27 following focal transient cerebral ischemia. Infarct volume, neurological deficit scores, physiological parameters, and immunohistochemical 
analyses were evaluated $24 \mathrm{~h}$ after reperfusion. Intravenous injections of hHSP27 $1 \mathrm{~h}$ after reperfusion significantly reduced infarct size and improved neurological deficits. Injected hHSP27 was localized in neurons on the ischemic side of the brain. hHSP27 suppressed neuronal cell death resulting from cytochrome c-mediated caspase activation, oxidative stress, and inflammatory responses. By contrast, recombinant HSP27 from Escherichia coli (E.coli), which was not phosphorylated, did not reduce infarct size or improve neurological deficits. Moreover, dephosphorylation of hHSP27 reduced its ability to protect the brain against ischemia. Therefore, phosphorylation of HSP27 was important in our experimental stroke models. We then determined whether in vitro phosphorylated recombinant HSP27 (prHSP27) protected brain against ischemic injury ${ }^{26}$. Mitogen-activated protein kinase-activated protein (MAPKA) kinase 2 phosphorylated the Ser15, Ser78, and Ser82 positions of rHSP27 in vitro. prHSP27 tended to occur as low molecular weight dimers or tetramers rather than high molecular weight oligomers. We also examined the possibility that the $\alpha \mathrm{B}-$ crystallin and HSP20 parts of the hHSP27 complex influence its effects on brain protection. Previous studies showed that due to similarities in structure and other properties, HSP27, $\alpha \mathrm{B}^{-}$-crystallin, and HSP20 are co-purified ${ }^{24)}$; moreover, HSP20 and $\alpha \mathrm{B}^{-}$crystallin are also neuroprotective ${ }^{28)}{ }^{29)}$. To test this, we examined how intravenous injection of $\alpha \mathrm{B}$-crystallin, HSP20, or prHSP27 with $\alpha \mathrm{B}$-crystallin and HSP20 affected brain protection. We did not observe additional neuro-protective effects of prHSP27 when combined with HSP20 and $\alpha \mathrm{B}^{- \text {crys- }}$ tallin compared to prHSP27 alone. prHSP27 injected into the tail veins of mice $1 \mathrm{~h}$ following reperfusion from ischemia appeared in serum at concentrations of $22.94 \pm 2.39 \mu \mathrm{g} / \mathrm{ml}$ after $15 \mathrm{~min}$ and $2.56 \pm 1.27$ $\mu \mathrm{g} / \mathrm{m} l$ after $60 \mathrm{~min}$. prHSP27 was not detected at $6 \mathrm{~h}, 12 \mathrm{~h}$, or $24 \mathrm{~h}$ after the injection. Although prHSP27 disappeared rather rapidly from plasma, it might remain in various other tissues. To obtain direct evidence of prHSP27 localization in the brain, we injected FITC-prHSP27 and microscopically examined fresh frozen sections of brain tissue. FITC-prHSP27 was diffusely localized inside neurons, glia, and endothelial cells in the brain. Thus, injected HSP27 might exert its effects both intra- and extracellularly.

prHSP27 also protected the BBB. The BBB is important for regulating the neural interface with the brain vasculature; whereby it regulates the movement of ions, molecules, and cells between neural cells and the blood, and controls ionic homeostasis, hormonal and transmitter levels, and the transport of nutrients in the brain ${ }^{30}$. The $\mathrm{BBB}$ is formed mainly by brain capillary endothelial cells, pericytes, and astrocytes. Ischemic insult caused overexpression of matrix metalloproteinases (MMPs) and injured the BBB, thereby increasing its permeability. The disrupted BBB expanded brain injury. Tissue plasminogen activator ( $\mathrm{tPA}$ ) is the only effective fibrinolytic treatment at the acute stage of ischemic stroke ${ }^{31}$; however, it is associated with an increased risk of brain edema and secondary hemorrhagic transformation. Disruption of the BBB has been shown to involve edema and hemorrhagic transformation after treatment of ischemic stroke. Thus, both ischemic injury and tPA treatment injure the $\mathrm{BBB}$, indicating that patients with ischemic stroke treated with tPA require drugs to protect the $\mathrm{BBB}$. Currently, only edaravone, a potent free-radical scavenger, is available for $\mathrm{BBB}$ protection ${ }^{32}$; however, it has limited use due to its side effects, including liver and renal dysfunction ${ }^{33}$. While prHSP27 protected both brain and BBB, co-administration of prHSP27 and tPA might be a good combination therapy. PrHSP27 might attenuate brain damage and prevented BBB disruption and hemorrhage caused by tPA.

Our ultimate goal is to complete the studies necessary to bring HSP27 protein therapy to the clinical level (Figure-1). We have reconfirmed the effect of HSP27 injection therapy in transient middle cerebral artery occlusion model mice which is ischemic stroke model. We are currently examining the prHSP27-protection of brain in different stroke models, for example in rat permanent middle cerebral artery occlusion. The $2 \mathrm{mg} / \mathrm{kg}$-dose of prHSP27 needed for neuroprotection in our model appears to be sufficient for its protective effects in patients with stroke and we injected it intravenously, which should be the same administration route used clinically. Moreover, human equivalent doses are lower and may be clinically feasible, although further studies are needed to establish them (Guidance for Industry Estimating the 


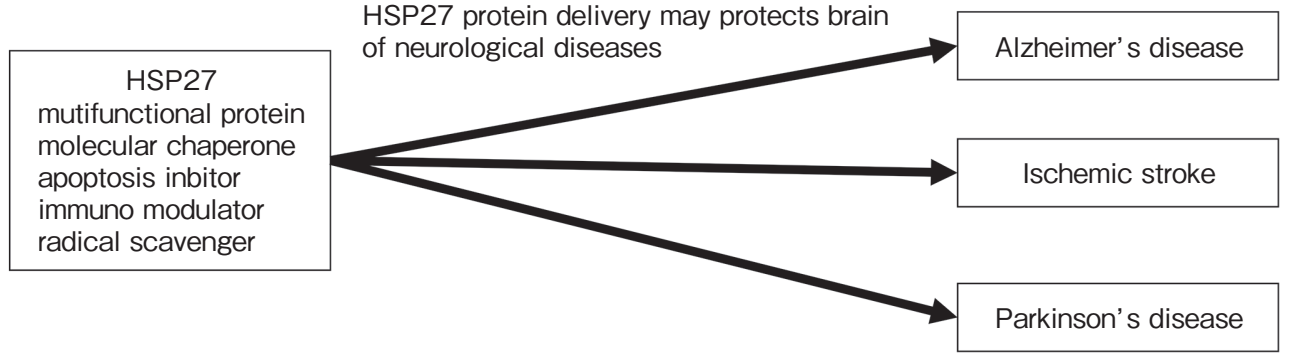

Figure-1

Injection of HSP protein might protect brain of Parkinson's disease, Alzheimer's disease and ischemic stroke.

Maximum Safe Starting Dose in Initial Clinical Trials for Therapeutics in Adult Healthy Volunteers, http://www.fda.gov/downloads/Drugs/ Guidances/UCM078932. pdf\#search=\%27guidekines +for+industry+sfe+starting\%27). We have not yet observed any severe adverse effects of HSP27. What remains is to develop HSP27 injection therapy for patients with stroke. If and when HSP27 injection therapy is established for ischemic stroke, it may also prove useful for other diseases as well, including Alzheimer's disease, Parkinson's disease, atherosclerotic disease, ischemic heart disease, and ischemic renal disease.

\section{Reference}

1) Mymrikov EV, Seit-Nebi AS, Gusev NB: Large potentials of small heat shock proteins. Physiol Rev, 2011; 91: 1123-1159.

2) Sugiyama Y, Suzuki A, Kishikawa M, et al: Muscle develops a specific form of small heat shock protein complex composed of MKBP/HSPB2 and HSPB3 during myogenic differentiation. J Biol Chem, 2000; 275: 1095-1104

3) Moran L, Mirault ME, Arrigo AP, GoldschmidtClermont M, Tissières A: Heat shock of Drosophila melanogaster induces the synthesis of new messenger RNAs and proteins. Philos Trans R Soc Lond B Biol Sci, 1978; 283: 391-406.

4) Jakob U, Gaestel M, Engel K, Buchner J: Small heat shock proteins are molecular chaperones. J Biol Chem, 1993; 268: 1517-1520.

5) Arrigo AP, Virot S, Chaufour S, Firdaus W, Kretz-Remy C, Diaz-Latoud C: Hsp27 consolidates intracellular redox homeostasis by upholding glutathione in its reduced form and by decreasing iron intracellular levels. Antioxid Redox Signal, 2005; 7: 414-422.

6) Charette SJ, Landry J: The interaction of HSP27 with Daxx identifies a potential regulatory role of HSP27 in Fas-induced apoptosis. Ann N Y Acad Sci, 2000; 926: 126-131.

7) Bruey JM, Ducasse C, Bonniaud P, et al: Hsp27 negatively regulates cell death by interacting with cytochrome c. Nat Cell Biol, 2000; 2: 645-652.

8) Calderwood SK, Khaleque MA, Sawyer DB, Ciocca DR: Heat shock proteins in cancer: chaperones of tumorigenesis. Trends Biochem Sci, 2006; 31: 164-172.

9) Benndorf R, Hayess K, Ryazantsev S, Wieske M, Behlke J, Lutsch G: Phosphorylation and supramolecular organization of murine small heat shock protein HSP25 abolish its actin polymerization-inhibiting activity. J Biol Chem, 1994; 269: 20780-20784.

10) Batulan Z, Pulakazhi Venu VK, Li Y, et al: Extracellular release and signaling by heat shock protein 27: role in modifying vascular inflammation. Front Immunol, 2016; 7: 285.

11) Vidyasagar A, Wilson NA, Djamali A: Heat shock protein 27 (HSP27): biomarker of disease and therapeutic target. Fibrogenesis Tissue Repair 2012; 5: 7.

12) Nemes Z, Devreese B, Steinert PM, Van Beeumen J, Fésüs L: Cross-linking of ubiquitin, HSP27, parkin, and alpha-synuclein by gamma-glutamyl-epsilon-lysine bonds in Alzheimer's neurofibrillary tangles. FASEB J, 2004; 18: 1135-1137.

13) Shinohara H, Inaguma $Y$, Goto S, Inagaki T, Kato K: Alpha B crystallin and HSP28 are enhanced in the cerebral cortex of patients with Alzheimer's disease. J Neurol Sci, 1993; 119: 203-208.

14) Shimura H, Miura-Shimura Y, Kosik KS: Binding of tau to heat shock protein 27 leads to decreased concentration of hyperphosphorylated tau and enhanced cell survival. J Biol Chem, 2004; 279: 17957-17962.

15) Abisambra JF, Blair LJ, Hill SE, et al: Phosphorylation dynamics regulate Hsp27-mediated rescue of neuronal plasticity deficits in tau transgenic mice. J Neurosci, 2010; 30: 15374-15382.

16) Tóth ME, Szegedi V, Varga E, et al: Overexpression of Hsp27 ameliorates symptoms of Alzheimer's disease in APP/PS1 mice. Cell Stress Chaperones, 2013; 18: 759-771.

17) Outeiro TF, Klucken J, Strathearn KE, et al: Small heat shock proteins protect against alpha-synuclein-induced toxicity and aggregation. Biochem Biophys Res Commun, 2006; 351: 631-638.

18) McLean PJ, Kawamata H, Shariff S, et al: Torsin A and heat shock proteins act as molecular chaperones: suppression of alpha-synuclein aggregation. J Neurochem, 2002; 83: 846-854.

19) Cox D, Carver JA, Ecroyd H: Preventing $\alpha$-synuclein aggregation: the role of the small heat-shock molecular chaperone proteins. Biochim Biophys Acta, 2014; 1842: 1830-1843. 
20) Cox D, Selig E, Griffin MDW, Carver JA, Ecroyd H: Small heat shock proteins prevent alpha-synuclein aggregation via transient interactions and their efficacy is affected by the rate of aggregation. J Biol Chem 2016; 291: 22618-22629.

21) Phan TG, Wright PM, Markus R, Howells DW, Davis SM, Donnan GA: Salvaging the ischaemic penumbra: more than just reperfusion? Clin Exp Pharmacol Physiol, 2002; 29: $1-10$.

22) Hossmann KA: Pathophysiological basis of translational stroke research. Folia Neuropathol, 2009; 47: 213-227.

23) Warner DS, Sheng H, Batinić-Haberle I: Oxidants, antioxidants and the ischemic brain. J Exp Biol, 2004; 207: 3221-3231.

24) Kato H, Liu Y, Kogure K, Kato K: Induction of 27-kDa heat shock protein following cerebral ischemia in a rat model of ischemic tolerance. Brain Res, 1994; 634: $235-244$.

25) Stetler RA, Cao G, Gao Y, et al: Hsp27 protects against ischemic brain injury via attenuation of a novel stress-response cascade upstream of mitochondrial cell death signaling. J Neurosci, 2008; 28: 13038-13055.

26) Teramoto S, Shimura H, Tanaka $\mathrm{R}$, et al: Humanderived physiological heat shock protein 27 complex protects brain after focal cerebral ischemia in mice. PLoS One, 2013; 8: e66001.

27) Shimada Y, Tanaka R, Shimura H, Yamashiro K, Urabe $\mathrm{T}$, Hattori N: Phosphorylation enhances recombinant HSP27 neuroprotection against focal cerebral ischemia in mice. Neuroscience, 2014; 278: 113-121.

28) Li T, Mo X, Jiang $Z$, et al: Study of $\alpha \mathrm{B}$-crystallin expression in Gerbil BCAO model of transient global cerebral ischemia. Oxid Med Cell Longev, 2012; 2012: 945071

29) Zeng L, Tan J, Hu Z, Lu W, Yang B: Hsp20 protects neuroblastoma cells from ischemia/reperfusion injury by inhibition of apoptosis via a mechanism that involves the mitochondrial pathways. Curr Neurovasc Res, 2010; 7: 281-287.

30) Luissint AC, Artus C, Glacial F, Ganeshamoorthy K, Couraud PO: Tight junctions at the blood brain barrier: physiological architecture and disease-associated dysregulation. Fluids Barriers CNS, 2012; 9: 23.

31) Wardlaw JM, Murray V, Berge E, et al: Recombinant tissue plasminogen activator for acute ischaemic stroke: an updated systematic review and meta-analysis. Lancet, 2012; 379: 2364-2372.

32) Kawai H, Nakai H, Suga M, Yuki S, Watanabe T, Saito KI: Effects of a novel free radical scavenger, $\mathrm{MCl}-186$, on ischemic brain damage in the rat distal middle cerebral artery occlusion model. J Pharmacol Exp Ther, 1997; 281: 921-927.

33) Lapchak PA: A critical assessment of edaravone acute ischemic stroke efficacy trials: is edaravone an effective neuroprotective therapy? Expert Opin Pharmacother, 2010; 11: 1753-1763. 